\title{
Dynamics of a fractional order mathematical model for COVID-19 epidemic
}

\author{
Zizhen Zhang ${ }^{1}$, Anwar Zeb ${ }^{2 *}$, Oluwaseun Francis Egbelowo ${ }^{3}$ and Vedat Suat Erturk ${ }^{4}$
}

"Correspondence:

anwar@cuiatd.edu.pk

2Department of Mathematics, COMSATS University Islamabad, Abbottabad Campus, Abbottabad, 22060, Khyber Pakhtunkhwa, Pakistan

Full list of author information is available at the end of the article

\begin{abstract}
In this work, we formulate and analyze a new mathematical model for COVID-19 epidemic with isolated class in fractional order. This model is described by a system of fractional-order differential equations model and includes five classes, namely, $S$ (susceptible class), E (exposed class), I (infected class), Q (isolated class), and $R$ (recovered class). Dynamics and numerical approximations for the proposed fractional-order model are studied. Firstly, positivity and boundedness of the model are established. Secondly, the basic reproduction number of the model is calculated by using the next generation matrix approach. Then, asymptotic stability of the model is investigated. Lastly, we apply the adaptive predictor-corrector algorithm and fourth-order Runge-Kutta (RK4) method to simulate the proposed model. Consequently, a set of numerical simulations are performed to support the validity of the theoretical results. The numerical simulations indicate that there is a good agreement between theoretical results and numerical ones.
\end{abstract}

Keywords: COVID-19 epidemic; Stability analysis; Adaptive predictor-corrector algorithm; Fractional differential equations; Numerical simulations

\section{Introduction}

Mathematical models describing infectious diseases have an important role both in theory and practice (see, for example, $[6-8,18,34])$. The construction and analysis of models of this type can help us understand the mechanism of the transmission as well as characteristics of diseases, and therefore, we can propose effective strategies to predict, prevent, and restrain diseases, as well as to protect population health. Up to now, many mathematical models for infectious diseases formulated by differential equations have been constructed and analyzed to study the spreading of viruses, for instance, [6-8, 18, 34]. Recently, mathematical models for COVID-19 epidemic have attracted the attention of many mathematicians, biologists, epidemiologists, pharmacists, chemists with many notable and important results (see, for instance, [1, 5, 9, 14, 15, 19, 23, 26, 28-30, 33, 35, 36] and references therein). This can be considered an effective approach to study, simulate, and predict the mechanism and transmission of COVID-19.

Motivated by the above reason, in this work, we formulate and analyze a new mathematical model for COVID-19 epidemic. This model is described by a system of fractional-order differential equations model and includes five classes, namely, $S$ (susceptible class), $E$ (ex-

(c) The Author(s) 2020. This article is licensed under a Creative Commons Attribution 4.0 International License, which permits use sharing, adaptation, distribution and reproduction in any medium or format, as long as you give appropriate credit to the original author(s) and the source, provide a link to the Creative Commons licence, and indicate if changes were made. The images or other third party material in this article are included in the article's Creative Commons licence, unless indicated otherwise in a credit line to the material. If material is not included in the article's Creative Commons licence and your intended use is not permitted by statutory regulation or exceeds the permitted use, you will need to obtain permission directly from the copyright holder. To view a copy of this licence, visit http://creativecommons.org/licenses/by/4.0/. 
posed class), $I$ (infected class), $Q$ (isolated class), and $R$ (recovered class). This model is a generalization of a well-known ODE model formulated in [35]. In the proposed fractionalorder model, we use the Caputo fractional derivative instead of the integer-order one because when modeling processes and phenomena arising in the real world, fractional-order models are more accurate than integer-order ones. In particular, fractional-order models provide more degrees of freedom in the model while an unlimited memory is also guaranteed in contrast to integer-models with limited memory [11, 24, 27].

Our main aim is to study the dynamics and numerical approximations for the proposed fractional-order model. Firstly, the positivity and boundedness of the model are investigated based on standard techniques of mathematical analysis. Secondly, the basic reproduction number of the model is calculated by using the next generation matrix approach. Then, asymptotic stability of the model is investigated based on the Lyapunov stability theorem for fractional dynamical systems. Lastly, we apply the adaptive predictor-corrector algorithm and fourth-order Runge-Kutta (RK4) method formulated in [20] to simulate the proposed model. Consequently, a set of numerical simulations is performed to support the validity of the theoretical results. The numerical simulations indicate that there is a good agreement between theoretical results and numerical ones.

This work is organized as follows. The fractional-order differential model is formulated in Sect. 2. Dynamics of the model is investigated in Sect. 3. Numerical simulations by the adaptive predictor-corrector algorithm are performed in Sect. 4. The last section present some remarks, conclusions, and discussions.

\section{Model formulation}

The total population is divided into five compartments: susceptible $(S)$, exposed $(E)$, infected $(I)$, isolated $(Q)$, and recovered $(R)$ from the disease. We assume that all the classes are normalized. This leads to the mathematical model formulation in which the interaction of the exposed population and infected population is linked to the susceptible population. In this model, we assume that the natural death rate includes the disease death rate. When there is no symptom of the disease, the exposed class moves with a certain rate to the isolated class, but in the case when symptoms are developed, it moves to the infected class. Keeping in mind the above assumptions, we obtain the following ODE model (see [35]):

$$
\begin{aligned}
& \frac{d S(t)}{d t}=\Lambda-\mu S(t)-\beta S(t)(E(t)+I(t)) \\
& \frac{d E(t)}{d t}=\beta S(t)(E(t)+I(t))-\pi E(t)-(\mu+\gamma) E(t), \\
& \frac{d I(t)}{d t}=\pi E(t)-\sigma I(t)-\mu I(t), \\
& \frac{d Q(t)}{d t}=\gamma E(t)+\sigma I(t)-\theta Q(t)-\mu Q(t) \\
& \frac{d R(t)}{d t}=\theta Q(t)-\mu R(t)
\end{aligned}
$$

where the parameters and variables used are described in Table 1.

Let us introduce the notation

$$
N=S+E+I+Q+R
$$


Table 1 Parameters in the model

\begin{tabular}{ll}
\hline Symbols & Description \\
\hline$S$ & Susceptible population \\
$E$ & Exposed population \\
$I$ & Infected Population \\
$Q$ & Isolated population \\
$R$ & Recovered population \\
$\Lambda=\mu N$ & Recruitment rate \\
$\beta$ & Rate at which susceptible move to infected and exposed class \\
$\pi$ & Rate at which exposed population moves to infected one \\
$\gamma$ & Rate at which exposed people become isolated \\
$\sigma$ & Rate at which infected people are added to isolated individuals \\
$\theta$ & Rate at which isolated persons become recovered \\
$\mu$ & Natural death rate plus disease related death rate \\
\hline
\end{tabular}

From the ODE system (1), we obtain

$$
\frac{d N}{d t}=\Lambda-\mu N
$$

which implies that the total population in this case we take as constant because $\Lambda=\mu N$.

To include into the model (1) the past history or hereditary properties, we replace the first derivative by the Caputo fractional derivative. More precisely, we propose the following system of fractional differential equations:

$$
\begin{aligned}
& { }_{0}^{C} D_{t}^{\alpha} S=\Lambda^{\alpha}-\mu^{\alpha} S-\beta^{\alpha} S(E+I), \\
& { }_{0}^{C} D_{t}^{\alpha} E=\beta^{\alpha} S(E+I)-\pi^{\alpha} E-\left(\mu^{\alpha}+\gamma^{\alpha}\right) E, \\
& { }_{0}^{C} D_{t}^{\alpha} I=\pi^{\alpha} E-\sigma^{\alpha} I-\mu^{\alpha} I, \\
& { }_{0}^{C} D_{t}^{\alpha} Q=\gamma^{\alpha} E+\sigma^{\alpha} I-\theta^{\alpha} Q-\mu^{\alpha} Q, \\
& { }_{0}^{C} D_{t}^{\alpha} R=\theta^{\alpha} Q(t)-\mu^{\alpha} R(t),
\end{aligned}
$$

where $0<\alpha<1$, and ${ }_{0}^{C} D_{t}^{\alpha}$ denotes the fractional derivative in the Caputo sense. We refer the readers to $[2-4,10,25]$ for the definition of the fractional Caputo derivative.

\section{Dynamics of the fractional-order model}

\subsection{Positivity and boundedness}

Let us denote

$$
\mathbb{R}_{+}^{4}=\{(S, E, I, Q) \mid S, E, I, Q \geq 0\} .
$$

The following theorem is proved by using a generalized mean value theorem $[21,22]$ and a fractional comparison principle [16, Lemma 10].

Theorem 1 (Positivity and boundedness) Let $\left(S_{0}, E_{0}, I_{0}, Q_{0}\right)$ be any initial data belonging to $\mathbb{R}_{+}^{4}$ and $(S(t), E(t), I(t), Q(t))$ be the solution corresponding to the initial data. Then, the 
set $\mathbb{R}_{+}^{4}$ is a positively invariant set of the model (3). Furthermore, we have

$$
\begin{aligned}
& \limsup _{t \rightarrow \infty} S(t) \leq S_{\infty}:=\frac{\Lambda^{\alpha}}{\mu^{\alpha}}, \\
& \limsup _{t \rightarrow \infty} E(t) \leq E_{\infty}:=\frac{\Lambda^{\alpha}}{\pi^{\alpha}+\mu^{\alpha}+\gamma^{\alpha}}, \\
& \limsup _{t \rightarrow \infty} I(t) \leq I_{\infty}:=\frac{\pi^{\alpha} E_{\infty}}{\sigma^{\alpha}+\mu^{\alpha}}, \\
& \limsup _{t \rightarrow \infty} Q(t) \leq Q_{\infty}:=\frac{\gamma^{\alpha} E_{\infty}+\sigma^{\alpha} I_{\infty}}{\delta^{\alpha}+\mu^{\alpha}}, \\
& \limsup _{t \rightarrow \infty} R(t) \leq \frac{\theta^{\alpha}}{\mu^{\alpha}} .
\end{aligned}
$$

Proof First, it is easy to prove the existence and unique of solutions of the model thanks to results proved in [17].

For the model (3), we have

$$
\begin{aligned}
& \left.{ }_{0}^{C} D_{t}^{\alpha} S\right|_{S=0}=\Lambda^{\alpha}>0, \\
& \left.{ }_{0}^{C} D_{t}^{\alpha} E\right|_{E=0}=\beta^{\alpha} S I \geq 0, \\
& \left.{ }_{0}^{C} D_{t}^{\alpha} I\right|_{I=0}=\pi^{\alpha} E \geq 0, \\
& \left.{ }_{0}^{C} D_{t}^{\alpha} Q\right|_{Q=0}=\gamma^{\alpha} E+\sigma^{\alpha} I \geq 0 .
\end{aligned}
$$

By (5) and the generalized mean value theorem [21, 22], we deduce that $S(t), E(t), I(t)$, $Q(t) \geq 0$ for all $t \geq 0$.

From the first equation of the system (3), we obtain

$$
{ }_{0}^{C} D_{t}^{\alpha} S \leq \Lambda^{\alpha}-\mu^{\alpha} S .
$$

By using the fractional comparison principle, we have the first estimate of (4).

From the second equation of the system (3), we have

$$
{ }_{0}^{C} D_{t}^{\alpha}(S+E) \leq \Lambda^{\alpha}-\mu^{\alpha} S-\pi^{\alpha} E-\left(\mu^{\alpha}+\gamma^{\alpha}\right) E,
$$

which implies that

$$
\limsup _{t \rightarrow \infty}(S(t)+E(t)) \leq E_{\infty} .
$$

Consequently, we have the second estimate of (4).

From the third equation of the system (3), we get

$$
{ }_{0}^{C} D_{t}^{\alpha} I \leq \pi^{\alpha} E_{\infty}-\sigma^{\alpha} I-\mu^{\alpha} I
$$

for $t$ large enough. This implies the third estimate of (4).

From the fourth equation of the system (3), we have

$$
{ }_{0}^{C} D_{t}^{\alpha} Q \leq \gamma^{\alpha} E_{\infty}+\sigma^{\alpha} I_{\infty}-\theta^{\alpha} Q-\mu^{\alpha} Q,
$$

for $t$ large enough. From this we get the fourth estimate of (4). 
Finally, the fifth equation of (3) implies that

$$
{ }_{0}^{C} D_{t}^{\alpha} R \leq \theta^{\alpha} Q_{\infty}-\mu^{\alpha} R
$$

which implies the fifth estimate of (4). The proof is complete.

\subsection{Equilibria and the reproduction number}

Firstly, to find equilibria of the model (3), we consider the following algebraic system:

$$
\begin{aligned}
& \Lambda^{\alpha}-\mu^{\alpha} S-\beta^{\alpha} S(E+I)=0, \\
& \beta^{\alpha} S(E+I)-\pi^{\alpha} E-\left(\mu^{\alpha}+\gamma^{\alpha}\right) E=0, \\
& \pi^{\alpha} E-\sigma^{\alpha} I-\mu^{\alpha} I=0, \\
& \gamma^{\alpha} E+\sigma^{\alpha} I-\theta^{\alpha} Q-\mu^{\alpha} Q=0, \\
& \theta^{\alpha} Q-\mu^{\alpha} R=0 .
\end{aligned}
$$

By some algebraic manipulations, we obtain two solutions of the system (6) that are

$$
S_{0}=\frac{\Lambda^{\alpha}}{\mu^{\alpha}}, \quad E_{0}=0, \quad I_{0}=0, \quad E_{0}=0, \quad R_{0}=0,
$$

and

$$
\begin{aligned}
& S^{*}=\frac{\Lambda^{\alpha}-\left(\pi^{\alpha}+\mu^{\alpha}+\gamma^{\alpha}\right) E^{*}}{\mu^{\alpha}}, \quad I^{*}=\frac{\pi^{\alpha}}{\sigma^{\alpha}+\mu^{\alpha}} E^{*}, \\
& Q^{*}=\frac{\gamma^{\alpha} E^{*}+\sigma^{\alpha} I^{*}}{\theta^{\alpha}+\mu^{\alpha}}, \\
& E^{*}=\frac{\beta^{\alpha} \Lambda^{\alpha}\left(\pi^{\alpha}+\sigma^{\alpha}+\mu^{\alpha}\right)-\mu^{\alpha}\left(\mu^{\alpha}+\sigma^{\alpha}\right)\left(\pi^{\alpha}+\mu^{\alpha}+\gamma^{\alpha}\right)}{\beta^{\alpha}\left(\pi^{\alpha}+\mu^{\alpha}+\gamma^{\alpha}\right)\left(\pi^{\alpha}+\sigma^{\alpha}+\mu^{\alpha}\right)}, \quad R^{*}=\frac{\theta^{\alpha} Q^{*}}{\mu^{\alpha}} .
\end{aligned}
$$

Note that $S^{*}, E^{*}, I^{*}, Q^{*}$ and $R^{*}$ are positive if and only if

$$
\beta^{\alpha} \Lambda^{\alpha}\left(\pi^{\alpha}+\sigma^{\alpha}+\mu^{\alpha}\right)-\mu^{\alpha}\left(\mu^{\alpha}+\sigma^{\alpha}\right)\left(\pi^{\alpha}+\mu^{\alpha}+\gamma^{\alpha}\right)>0
$$

We now compute the reproduction number of the model (3) by using the next generation matrix approach developed by van den Driessche and Watmough [31]. Let $x=$ $(E, I, Q, R, S)$. We rewrite the model (3) in the matrix form

$$
{ }_{0}^{C} D_{t}^{\alpha} x=\mathcal{F}(x)-\mathcal{V}(x),
$$

where

$$
\mathcal{F}(x)=\left(\begin{array}{c}
\beta^{\alpha} S(E+I) \\
0 \\
0 \\
0 \\
0
\end{array}\right), \quad \mathcal{V}(x)=\left(\begin{array}{c}
\pi^{\alpha} E+\left(\mu^{\alpha}+\gamma^{\alpha}\right) E \\
-\pi^{\alpha} E+\sigma^{\alpha} I+\mu^{\alpha} I \\
-\gamma^{\alpha} E-\sigma^{\alpha} I+\theta^{\alpha} Q+\mu^{\alpha} Q \\
-\theta^{\alpha} Q+\mu^{\alpha} R \\
-\Lambda^{\alpha}+\mu^{\alpha} S+\beta^{\alpha} S(E+I)
\end{array}\right) \text {. }
$$


Hence, the reproduction number of the model (3) can be determined by

$$
\mathcal{R}_{0}=\rho\left(F V^{-1}\right)=\frac{\beta^{\alpha} \Lambda^{\alpha}\left(\pi^{\alpha}+\sigma^{\alpha}+\mu^{\alpha}\right)}{\mu^{\alpha}\left(\sigma^{\alpha}+\mu^{\alpha}\right)\left(\pi^{\alpha}+\mu^{\alpha}+\gamma^{\alpha}\right)} .
$$

It is easy to verify that $S^{*}, E^{*}, I^{*}, Q^{*}$, and $R^{*}>0$ if and only if $\mathcal{R}_{0}>1$.

Theorem 2 (Equilibria) The model (3) always possesses a disease-free equilibrium (DFE) point $F_{0}=\left(S_{0}, E_{0}, I_{0}, Q_{0}, R_{0}\right)$ given by (8) for all values of the parameters. Moreover, the model has a unique disease endemic equilibrium point $F^{*}=\left(S^{*}, E^{*}, I^{*}, Q^{*}, R^{*}\right)$ given by (9) if and only if $\mathcal{R}_{0}>1$.

\subsection{Stability analysis}

Theorem 3 The DFE point of the model (3) is locally asymptotically stable if $\mathcal{R}_{0}<1$.

Proof The Jacobian matrix of the model (3) at the DFE point is

$$
J\left(F_{0}\right)=\left(\begin{array}{ccccc}
-\mu^{\alpha} & -\beta^{\alpha} S_{0} & -\beta^{\alpha} S_{0} & 0 & 0 \\
0 & \beta^{\alpha} S_{0}-\left(\pi^{\alpha}+\mu^{\alpha}+\gamma^{\alpha}\right) & \beta^{\alpha} S_{0} & 0 & 0 \\
0 & \pi^{\alpha} & -\left(\sigma^{\alpha}+\mu^{\alpha}\right) & 0 & 0 \\
0 & \gamma^{\alpha} & \sigma^{\alpha} & -\left(\theta^{\alpha}+\mu^{\alpha}\right) & 0 \\
0 & 0 & 0 & \theta^{\alpha} & -\mu^{\alpha}
\end{array}\right) .
$$

The characteristic polynomial of $J$ is

$$
P_{J}(x)=\left(\lambda+\mu^{\alpha}\right)\left[\lambda+\left(\theta^{\alpha}+\mu^{\alpha}\right)\right]\left(\lambda+\mu^{\alpha}\right)\left(\lambda^{2}+\tau_{1} \lambda+\tau_{2}\right),
$$

where

$$
\begin{aligned}
& \tau_{1}=-\left[\beta S_{0}-\left(\pi^{\alpha}+\mu^{\alpha}+\gamma^{\alpha}\right)-\left(\sigma^{\alpha}+\mu^{\alpha}\right)\right], \\
& \tau_{2}=\left(\pi^{\alpha}+\mu^{\alpha}+\gamma^{\alpha}\right)\left(\sigma^{\alpha}+\mu^{\alpha}\right)-\beta^{\alpha} S_{0}\left(\pi^{\alpha}+\sigma^{\alpha}+\mu^{\alpha}\right) .
\end{aligned}
$$

It is easy to verify that if $\mathcal{R}_{0}<1$, then $\tau_{1}>0$ and $\tau_{2}>0$. This implies that two roots of the polynomial $\lambda^{2}+\tau_{1} \lambda+\tau_{2}$ have negative real parts. Consequently, the real parts of five eigenvalues of the matrix $J\left(F_{0}\right)$ are all negative, or equivalently, $F_{0}$ is locally stable. The proof is complete.

We now prove the uniform asymptotical stability of the DFE point of the model (3) by using the Lyapunov stability theorem [12, Theorem 3.1].

Theorem 4 If $\mathcal{R}_{0}<1$, then the DFE point of the model (3) is not only locally asymptotically stable but also uniformly asymptotically stable.

Proof Since the first three equations of the model (3) do not depend on the states $Q$ and $R$, we need only consider the following subsystem:

$$
\begin{aligned}
& { }_{0}^{C} D_{t}^{\alpha} S=\Lambda^{\alpha}-\mu^{\alpha} S-\beta^{\alpha} S(E+I), \\
& { }_{0}^{C} D_{t}^{\alpha} E=\beta^{\alpha} S(E+I)-\pi^{\alpha} E-\left(\mu^{\alpha}+\gamma^{\alpha}\right) E, \\
& { }_{0}^{C} D_{t}^{\alpha} I=\pi^{\alpha} E-\sigma^{\alpha} I-\mu^{\alpha} I .
\end{aligned}
$$


From (4), it is sufficient to consider the model (3) in its feasible set defined by

$$
\Omega=\left\{(S, E, I) \mid S, E, I \geq 0, S \leq \frac{\Lambda^{\alpha}}{\mu^{\alpha}}\right\} .
$$

Consider a Lyapunov function $V: \Omega \rightarrow \mathbb{R}_{+}$given by

$$
V(S, E, I)=\left(S-S_{0} \ln \frac{S}{S_{0}}-S_{0}\right)+E+\frac{\pi^{\alpha}+\mu^{\alpha}+\gamma^{\alpha}}{\pi^{\alpha}+\sigma^{\alpha}+\mu^{\alpha}} I .
$$

By using the linearity property of the Caputo derivative and [32, Lemma 3.1] and some algebraic manipulations, we obtain

$$
{ }_{0}^{C} D_{t}^{\alpha} V \leq-\left(S-S_{0}\right)^{2}+\tau_{1} I+\tau_{2} E,
$$

where

$$
\tau_{1}=\beta^{\alpha} \frac{\Lambda^{\alpha}}{\mu^{\alpha}}-\left(\pi^{\alpha}+\mu^{\alpha}+\gamma^{\alpha}\right)+\pi^{\alpha} \frac{\pi^{\alpha}+\mu^{\alpha}+\gamma^{\alpha}}{\pi^{\alpha}+\sigma^{\alpha}+\mu^{\alpha}}, \quad \tau_{2}=\beta^{\alpha} \frac{\Lambda^{\alpha}}{\mu^{\alpha}}-\frac{\pi^{\alpha}+\sigma^{\alpha}+\gamma^{\alpha}}{\pi^{\alpha}+\sigma^{\alpha}+\mu^{\alpha}} .
$$

It is easy to check that if $\mathcal{R}_{0}<1$, then $\tau_{1}, \tau_{2}<0$. Consequently, by the Lyapunov stability theorem for fractional dynamical systems, we deduce that $F_{0}$ is uniformly asymptotically stable. The proof is complete.

Remark 1 The analysis of stability of $F^{*}$ is an interesting mathematical problem, but in this work, we mainly focus on the case $\mathcal{R}_{0}<1$ to find an effective strategy to prevent the disease.

\section{4 $\mathcal{R}_{0}$ sensitivity analysis}

Theorem 3 suggests that we should control the parameters such that $\mathcal{R}_{0}<1$. This provides a good strategy to prevent and restrain the disease. More precisely, when $\mathcal{R}_{0}<1$, then

$$
\lim _{t \rightarrow \infty} S(t)=\frac{\Lambda^{\alpha}}{\mu^{\alpha}}, \quad \lim _{t \rightarrow \infty} E(t)=\lim _{t \rightarrow \infty} I(t)=\lim _{t \rightarrow \infty} Q(t)=\lim _{t \rightarrow \infty} R(t)=0,
$$

which means that the disease will be completely controlled and prevented. Motivated by this, we now perform an $\mathcal{R}_{0}$ sensitivity analysis to find ways to choose suitable parameters.

It is easy to verify that

$$
\begin{aligned}
& \frac{\partial \mathcal{R}_{0}}{\partial \beta}=\frac{\alpha \beta^{\alpha-1} \Lambda^{\alpha}\left(\pi^{\alpha}+\sigma^{\alpha}+\mu^{\alpha}\right)}{\mu^{\alpha}\left(\sigma^{\alpha}+\mu^{\alpha}\right)\left(\pi^{\alpha}+\mu^{\alpha}+\gamma^{\alpha}\right)}>0, \\
& \frac{\partial \mathcal{R}_{0}}{\partial \pi}=\frac{\beta^{\alpha} \Lambda^{\alpha}}{\mu^{\alpha}\left(\sigma^{\alpha}+\mu^{\alpha}\right)} \frac{\alpha \pi^{\alpha-1}\left(\gamma^{\alpha}-\mu^{\alpha}\right)}{\left(\pi^{\alpha}+\mu^{\alpha}+\gamma^{\alpha}\right)^{2}}, \\
& \frac{\partial \mathcal{R}_{0}}{\partial \sigma}=-\frac{\beta^{\alpha} \Lambda^{\alpha}}{\mu^{\alpha}\left(\mu^{\alpha}+\mu^{\alpha}+\gamma^{\alpha}\right)} \frac{\alpha \sigma^{\alpha-1} \pi^{\alpha}}{\left(\sigma^{\alpha}+\mu^{\alpha}\right)^{2}}<0, \\
& \frac{\partial \mathcal{R}_{0}}{\partial \gamma}=-\frac{\beta^{\alpha} \Lambda^{\alpha}\left(\pi^{\alpha}+\sigma^{\alpha}+\mu^{\alpha}\right)}{\mu^{\alpha}\left(\sigma^{\alpha}+\mu^{\alpha}\right)} \frac{\alpha \gamma^{\alpha-1}}{\left(\pi^{\alpha}+\mu^{\alpha}+\gamma^{\alpha}\right)}<0 .
\end{aligned}
$$

Equation (16) suggests some ways to choose the parameters such that $\mathcal{R}_{0}<1$. Hence, based on this, we can propose suitable strategies to control and prevent the disease. 
4 Numerical simulations by the adaptive predictor-corrector algorithm

\subsection{The adaptive predictor-corrector algorithm}

In this section we review the method that is proposed by [20]. The proposed algorithm is given as follows. Consider the initial value problem (IVP) governed by:

$$
\begin{cases}D_{a+}^{\alpha, \rho} y(t)=f(t, y(t)), & t \in[0, T], \\ y^{k}(a)=y_{0}^{k}, & k=0,1,2, \ldots,[\alpha]-1,\end{cases}
$$

where $D_{a_{+}}^{\alpha, \rho}$ is the proposed generalized Caputo-type fractional derivative operator given [20, Definition 4]. Initially, for $m-1<\alpha \leq m, a \geq 0, \rho>0$ and $y \in C^{m}([a, T])$, the IVP (17) is equivalent, using [20, Theorem 3], to the Volterra integral equation:

$$
y(t)=u(t)+\frac{\rho^{1-\alpha}}{\Gamma(\alpha)} \int_{a}^{t}(s)^{\rho-1}\left(t^{\rho}-s^{\rho}\right)^{\alpha-1} f(s, y(s)) d s,
$$

where

$$
u(t)=\left.\sum_{n=0}^{m-1} \frac{1}{\rho^{n} n !}\left(t^{\rho}-a^{\rho}\right)^{n}\left[\left(x^{1-\rho} \frac{d}{d x}\right)^{n} y(x)\right]\right|_{x=a} .
$$

The first step of our algorithm, under the assumption that the function $f$ is such that a unique solution exists on some interval $[a, T]$, consists of dividing the interval $[a, T]$ into $N$ unequal subintervals $\left[t_{k}, t_{k+1}\right], k=0,1, \ldots, N-1$ using the mesh points

$$
\left\{\begin{array}{l}
t_{0}=a, \\
t_{k+1}=\left(t_{k}^{\rho}+h\right)^{\frac{1}{\rho}} \quad k=0,1,2, \ldots, N-1,
\end{array}\right.
$$

where $h=\frac{\left(T^{\rho}-a^{\rho}\right)}{N}$. Now, we are going to generate the approximations $y_{k}, k=0,1, \ldots, N$, to solve numerically the IVP (17). The basic step, assuming that we have already evaluated the approximations $y_{i} \approx y\left(t_{j}\right)(j=1,2, \ldots, k)$, is that we want to get the approximation $y_{k} \approx$ $y\left(t_{k+1}\right)$ by means of the integral equation

$$
y\left(t_{k+1}\right)=u\left(t_{k+1}\right)+\frac{\rho^{-\alpha}}{\Gamma(\alpha)} \int_{a}^{t_{k+1}} s^{\rho-1}\left(t_{k+1}^{\rho}-s^{\rho}\right)^{\alpha-1} f(s, y(s)) d s .
$$

Making the substitution

$$
z=(s)^{\rho}
$$

we get

$$
y\left(t_{k+1}\right)=u\left(t_{k+1}\right)+\frac{\rho^{-\alpha}}{\Gamma(\alpha)} \int_{a}^{t_{k+1}^{\rho}}\left(t_{k+1}^{\rho}-z\right)^{\alpha-1} f\left(z^{\frac{1}{\rho}}, y\left(z^{\frac{1}{\rho}}\right) d z,\right.
$$

that is,

$$
y\left(t_{k+1}\right)=u\left(t_{k+1}\right)+\frac{\rho^{-\alpha}}{\Gamma(\alpha)} \sum_{j=0}^{k} \int_{t_{j}^{\rho}}^{t_{j+1}^{\rho}}\left(t_{k+1}^{\rho}-z\right)^{\alpha-1} f\left(z^{\frac{1}{\rho}}, y\left(z^{\frac{1}{\rho}}\right) d z .\right.
$$


Next, if we use the trapezoidal quadrature rule with respect to the weight function $\left(t_{k+1}^{\rho}-\right.$ $z)^{\alpha-1}$ to approximate the integrals appear in the right-hand side of Eq. (24), replacing the function $f\left(z^{\frac{1}{\rho}}, y\left(z^{\frac{1}{\rho}}\right)\right)$ by its piecewise linear interpolant with nodes chosen at the $t_{j}^{\rho}(j=$ $0,1, \ldots, k+1)$, then we obtain

$$
\begin{aligned}
& \int_{t_{j}^{\rho}}^{t_{j+1}^{\rho}}\left(t_{k+1}^{\rho}-z\right)^{\alpha-1} f\left(z^{\frac{1}{\rho}}, y\left(z^{\frac{1}{\rho}}\right) d z\right. \\
& \approx \\
& \quad \frac{h^{\alpha}}{\alpha(\alpha+1)}\left[\left((k-j)^{\alpha+1}-(k-j-\alpha+)(k-j+1)^{\alpha}\right) f\left(t_{j}, y\left(t_{j}\right)\right)\right. \\
& \left.\quad+\left((k-j+1)^{\alpha+1}-(k-j+\alpha+1)(k-j)^{\alpha}\right) f\left(t_{j+1}, y\left(t_{j+1}\right)\right)\right] .
\end{aligned}
$$

Thus, substituting the above approximations into Eq. (24), we obtain the corrector formula for $y\left(t_{k+1}\right), k=0,1, \ldots, N-1$,

$$
y\left(t_{k+1}\right) \approx u\left(t_{k+1}\right)+\frac{\rho^{-\alpha}}{\Gamma(\alpha+2)} \sum_{j=0}^{k} a_{j, k+1} f\left(t_{j}, y\left(t_{j}\right)\right)+\frac{\rho^{-\alpha} h^{\alpha}}{\Gamma(\alpha+2)} f\left(t_{k+1}, y\left(t_{k+1}\right)\right),
$$

where

$$
a_{j, k+1}=\left\{\begin{array}{l}
k^{\alpha+1}-(k-\alpha)(k+1)^{\alpha} \quad \text { if } j=0, \\
(k-j+2)^{\alpha+1}+(k-j)^{\alpha+1}-2(k-j+1)^{\alpha+1} \quad \text { if } 1 \leq j<k .
\end{array}\right.
$$

The last step of our algorithm is to replace the quantity $y\left(t_{k+1}\right)$ shown on the right-hand side of formula (26) with the predictor value $y^{p}\left(t_{k+1}\right)$ that can be obtained by applying the one-step Adams-Bashforth method to the integral equation (23). In this case, by replacing the function $f\left(z^{\frac{1}{\rho}}, y\left(z^{\frac{1}{\rho}}\right)\right)$ with the quantity $f\left(t_{j}, y\left(t_{j}\right)\right)$ at each integral in Eq. (24), we get

$$
\begin{aligned}
y^{p}\left(t_{k+1}\right) & \approx u\left(t_{k+1}\right)+\frac{\rho^{-\alpha}}{\Gamma(\alpha)} \sum_{j=0}^{k} \int_{t_{j}^{\rho}}^{t_{j+1}^{\rho}}\left(t_{k+1}^{\rho}-z\right)^{\alpha-1} f\left(t_{j}, y\left(t_{j}\right)\right) d z \\
& =u\left(t_{k+1}\right)+\frac{\rho^{-\alpha} h^{\alpha}}{\Gamma(\alpha+1)} \sum_{j=0}^{k}\left[(k+1-j)^{\alpha}-(k-j)^{\alpha}\right] f\left(t_{j}, y\left(t_{j}\right)\right) .
\end{aligned}
$$

Therefore, the present adaptive predictor-corrector algorithm, for evaluating the approximation $y_{k+1} \approx y\left(t_{k+1}\right)$, is completely described by the formula

$$
y_{k+1} \approx u\left(t_{k+1}\right)+\frac{\rho^{-\alpha} h^{\alpha}}{\Gamma(\alpha+2)} \sum_{j=0}^{k} a_{j, k+1} f\left(t_{j}, y_{j}\right)+\frac{\rho^{-\alpha} h^{\alpha}}{\Gamma(\alpha+2)} f\left(t_{k+1}, y_{k+1}^{p}\right),
$$

where $y_{j} \approx y\left(t_{j}\right), j=0,1, \ldots, k$, and the predicted value $y_{k+1}^{p} \approx y^{p}\left(t_{k+1}\right)$ can be determined as described in Eq. (28) with the weights $a_{j, k+1}$ being defined according to (27). It is easily observed that when $\rho=1$, the present adaptive predictor-corrector algorithm will be reduced to the predictor-corrector approach presented in [13]. 


\subsection{Implementation with numerical simulation}

In this section, we solve numerically Eq. (3) using the method given in the former section. In view of the above algorithm, following the rule (27), the approximations $S_{k+1}, E_{k+1}, I_{k+1}$, $Q_{k+1}$, and $R_{k+1}$ can be simply evaluated using the iterative formulas, for $N \in \mathbb{N}$ and $T>0$,

$$
\begin{aligned}
S_{k+1} \approx & S_{0}+\frac{\rho^{-\alpha} h^{\alpha}}{\Gamma(\alpha+2)} \sum_{j=0}^{k} a_{j, k+1}\left[\Lambda^{\alpha}-\mu^{\alpha} S_{j}-\beta^{\alpha} S_{j}\left(E_{j}+I_{j}\right)\right] \\
& \left.+\frac{\rho^{-\alpha} h^{\alpha}}{\Gamma(\alpha+2)}\left[A^{\alpha}-\mu^{\alpha} S_{k+1}^{p}\right)-\beta^{\alpha} S_{k+1}^{p}\left(E_{k+1}^{p}+I_{k+1}^{p}\right)\right], \\
E_{k+1} \approx & E_{0}+\frac{\rho^{-\alpha} h^{\alpha}}{\Gamma(\alpha+2)} \sum_{j=0}^{k} a_{j, k+1}\left[\beta^{\alpha} S_{j}\left(E_{j}+I_{j}\right)-\left(\pi^{\alpha}+\mu^{\alpha}+\gamma^{\alpha}\right) E_{j}\right] \\
& +\frac{\rho^{-\alpha} h^{\alpha}}{\Gamma(\alpha+2)}\left[\beta^{\alpha} S_{k+1}^{p}\left(E_{k+1}^{p}+I_{k+1}^{p}\right)-\left(\pi^{\alpha}+\mu^{\alpha}+\gamma^{\alpha}\right) E_{k+1}^{p}\right], \\
I_{k+1} \approx & I_{0}+\frac{\rho^{-\alpha} h^{\alpha}}{\Gamma(\alpha+2)} \sum_{j=0}^{k} a_{j, k+1}\left[\pi^{\alpha} E_{j}-\left(\sigma^{\alpha}+\mu^{\alpha}\right) I_{j}\right] \\
& +\frac{\rho^{-\alpha} h^{\alpha}}{\Gamma(\alpha+2)}\left[\pi^{\alpha} E_{k+1}^{p}-\left(\sigma^{\alpha}+\mu^{\alpha}\right) I_{k+1}^{p}\right], \\
Q_{k+1} \approx & Q_{0}+\frac{\rho^{-\alpha} h^{\alpha}}{\Gamma(\alpha+2)} \sum_{j=0}^{k} a_{j, k+1}\left[\gamma^{\alpha} E_{j}+\sigma^{\alpha} I_{j}-\left(\theta^{\alpha}+\mu^{\alpha}\right) Q_{j}\right] \\
& +\frac{\rho^{-\alpha} h^{\alpha}}{\Gamma(\alpha+2)}\left[\gamma^{\alpha} E_{k+1}^{p}+\sigma^{\alpha} I_{k+1}^{p}-\left(\theta^{\alpha}+\mu^{\alpha}\right) Q_{k+1}^{p}\right], \\
& +\frac{\rho^{-\alpha} h^{\alpha}}{\Gamma(\alpha+2)}\left[\theta^{\alpha} Q_{k+1}^{p}-\mu^{\alpha} R_{k+1}^{p}\right], \\
R_{k+1} \approx & R_{0}+\frac{\rho^{-\alpha} h^{\alpha}}{\Gamma(\alpha+2)} \sum_{j=0}^{k} a_{j, k+1}\left[\theta^{\alpha} Q_{j}-\mu^{\alpha} R_{j}\right]
\end{aligned}
$$

where $h=\frac{T^{\rho}}{N}$ and

$$
\begin{aligned}
& S_{k+1}^{p} \approx S_{0}+\frac{\rho^{-\alpha} h^{\alpha}}{\Gamma(\alpha+1)} \sum_{j=0}^{k}\left((k+1-j)^{\alpha}-(k-j)^{\alpha}\right)\left[\Lambda^{\alpha}-\mu^{\alpha} S_{j}-\beta^{\alpha} S_{j}\left(E_{j}+I_{j}\right)\right] \\
& E_{k+1}^{p} \approx E_{0}+\frac{\rho^{-\alpha} h^{\alpha}}{\Gamma(\alpha+2)} \sum_{j=0}^{k}\left((k+1-j)^{\alpha}-(k-j)^{\alpha}\right)\left[\beta^{\alpha} S_{j}\left(E_{j}+I_{j}\right)-\left(\pi^{\alpha}+\mu^{\alpha}+\gamma^{\alpha}\right) E_{j}\right], \\
& I_{k+1}^{p} \approx I_{0}+\frac{\rho^{-\alpha} h^{\alpha}}{\Gamma(\alpha+2)} \sum_{j=0}^{k}\left((k+1-j)^{\alpha}-(k-j)^{\alpha}\right)\left[\pi^{\alpha} E_{j}-\left(\sigma^{\alpha}+\mu^{\alpha}\right) I_{j}\right], \\
& Q_{k+1}^{p} \approx Q_{0}+\frac{\rho^{-\alpha} h^{\alpha}}{\Gamma(\alpha+2)} \sum_{j=0}^{k}\left((k+1-j)^{\alpha}-(k-j)^{\alpha}\right)\left[\gamma^{\alpha} E_{j}+\sigma^{\alpha} I_{j}-\left(\theta^{\alpha}+\mu^{\alpha}\right) Q_{j}\right], \\
& R_{k+1}^{p} \approx R_{0}+\frac{\rho^{-\alpha} h^{\alpha}}{\Gamma(\alpha+2)} \sum_{j=0}^{k}\left((k+1-j)^{\alpha}-(k-j)^{\alpha}\right)\left[\theta^{\alpha} Q_{j}-\mu^{\alpha} R_{j}\right] .
\end{aligned}
$$




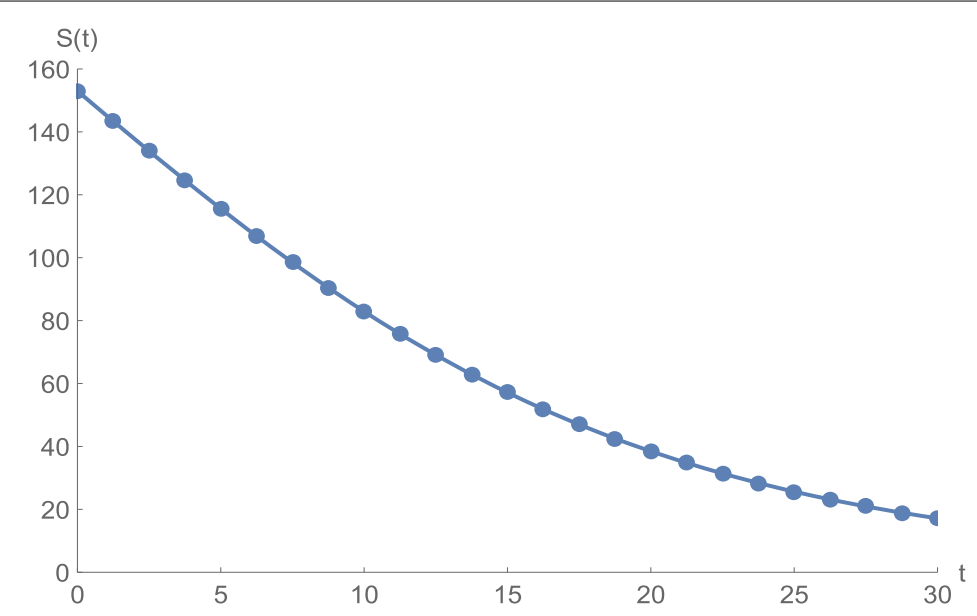

Figure $1 S(t)$ against $t$ : solid line presents the proposed approximation, dotted line stands for RK4 method

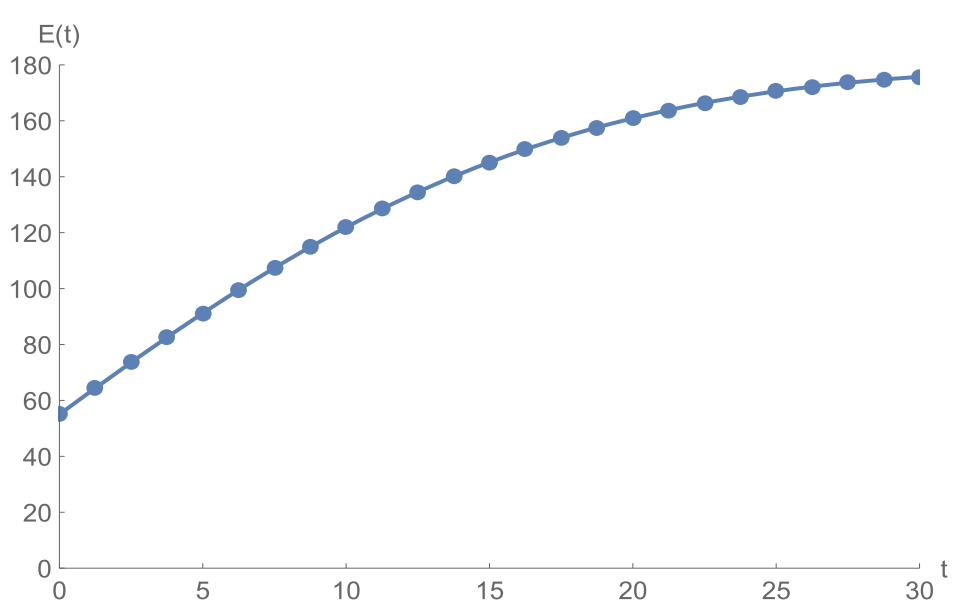

Figure $2 E(t)$ versus $t$ : solid line presents the proposed approximation, dotted line stands for RK4 method

Parameter values which are calculated for basic time of coronavirus used in numerical example are $\Lambda=0.145 ; \mu=0.000411 ; \beta=0.00038 ; \pi=0.00211 ; \gamma=0.0021 ; \sigma=$ $0.0169 ; \theta=0.0181$, with total population $N=355$ and initial data $\left(S_{0}, E_{0}, I_{0}, Q_{0}, R_{0}\right)=$ $(153,55,79,68,20)$.

In Figs. 1-5, we plot numerical solutions of the model (3) with $T=30$ obtained using the proposed algorithm and the RK4 method when $N=355$ and $\left(S_{0}, E_{0}, I_{0}, Q_{0}, R_{0}\right)=$ $(153,55,79,68,20)$ for $\alpha=1$. From the graphical results in Figs. $1-5$, it can be seen that the results obtained using the proposed algorithm match the results of the RK4 method very well, which implies that the presented method can predict the behavior of these variables accurately in the region under consideration.

Figures 6-10 show the approximate solutions for $S(t), E(t), I(t), Q(t)$, and $R(t)$ obtained for different values of $\alpha$ using the proposed algorithm. From the graphical results given in Figs. 6-10, it is clear that the approximate solutions depend continuously on the timefractional derivative $\alpha$. It is evident that the efficiency of this approach can be dramatically enhanced by decreasing the step size. 


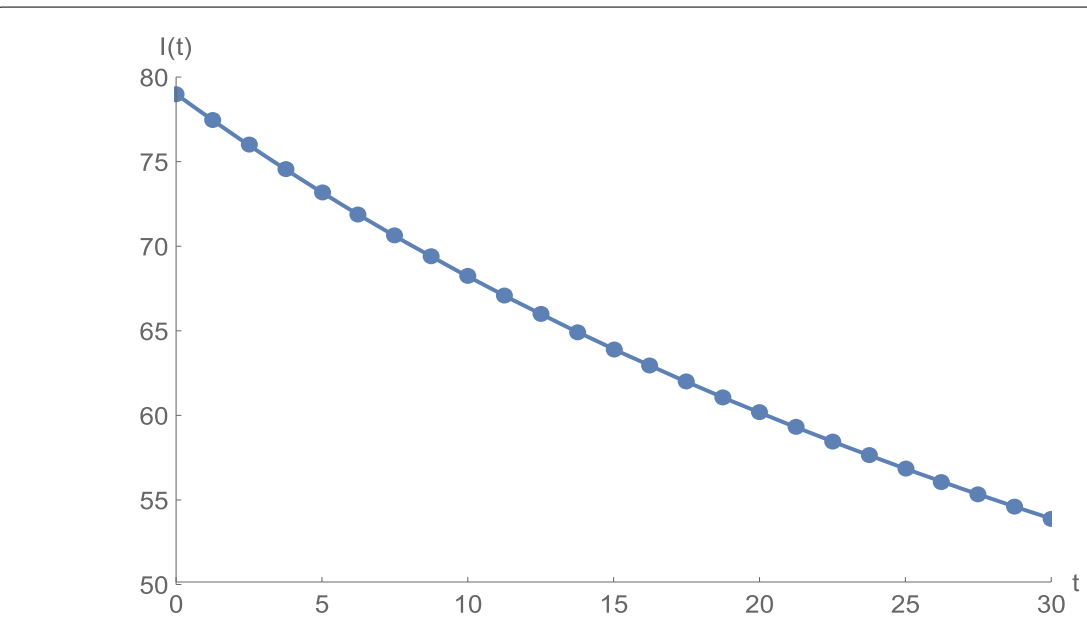

Figure $3 /(t)$ against $t$ : solid line presents the proposed approximation, dotted line stands for RK4 method

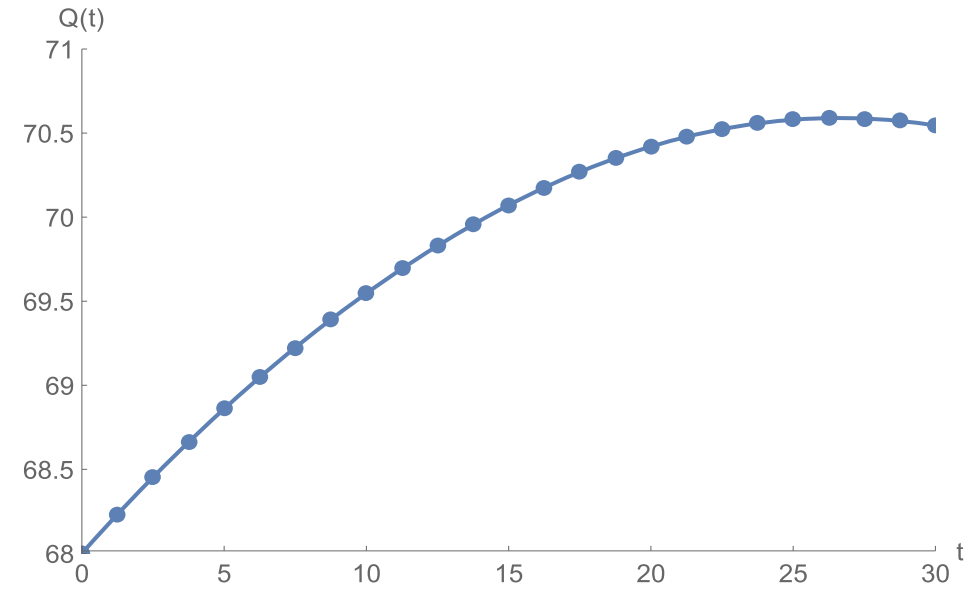

Figure $4 Q(t)$ against $t$ : solid line presents the proposed approximation, dotted line stands for RK4 method

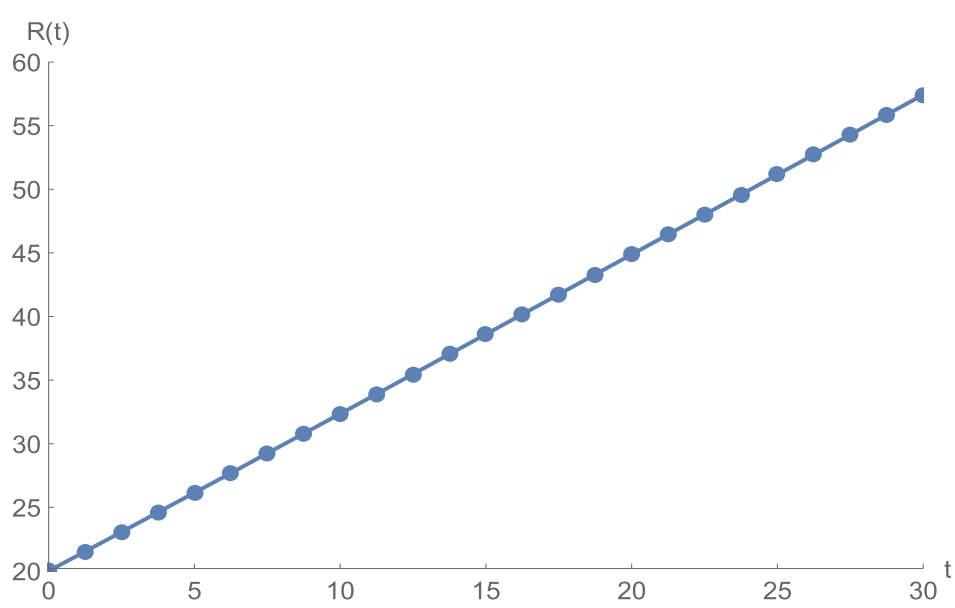

Figure $5 R(t)$ against $t$ : solid line presents the proposed approximation, dotted line stands for RK4 method 


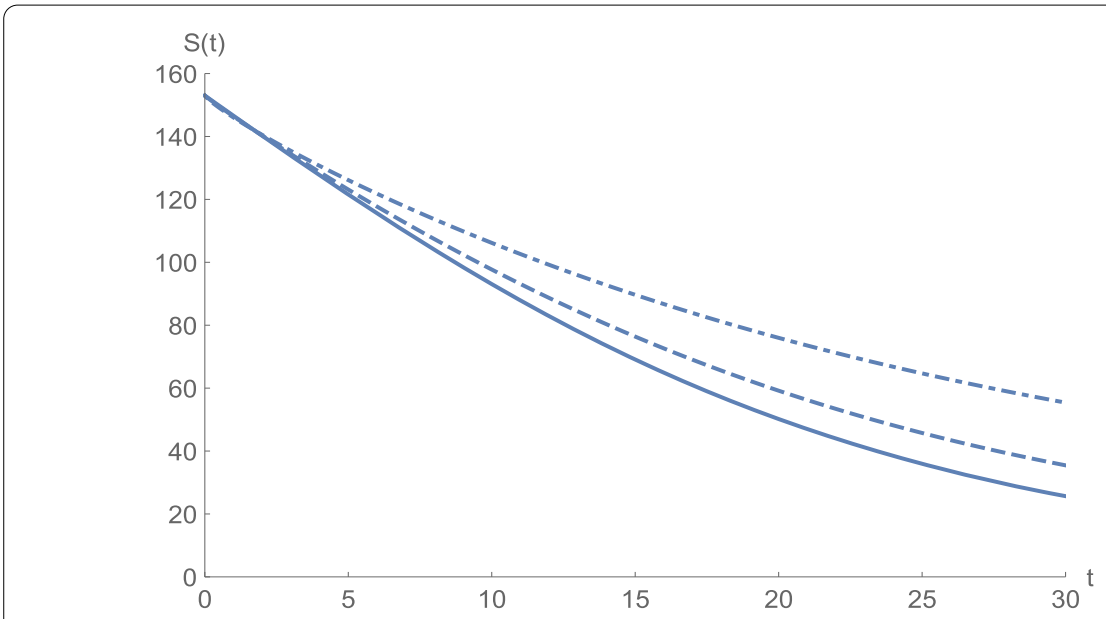

Figure $6 \mathrm{~S}(t)$ against $t$ : (solid line) $a=1.0$, (dot-dashed line) $a=0.85$, (dashed line) $a=0.75$

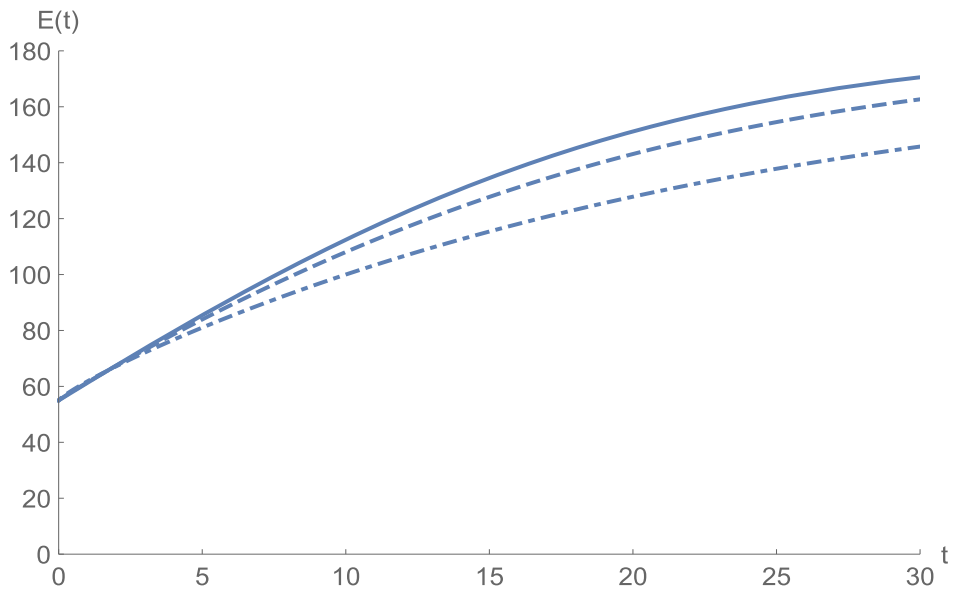

Figure $7 E(t)$ against $t$ : (solid line) $a=1.0$, (dot-dashed line) $a=0.85$, (dashed line) $a=0.75$

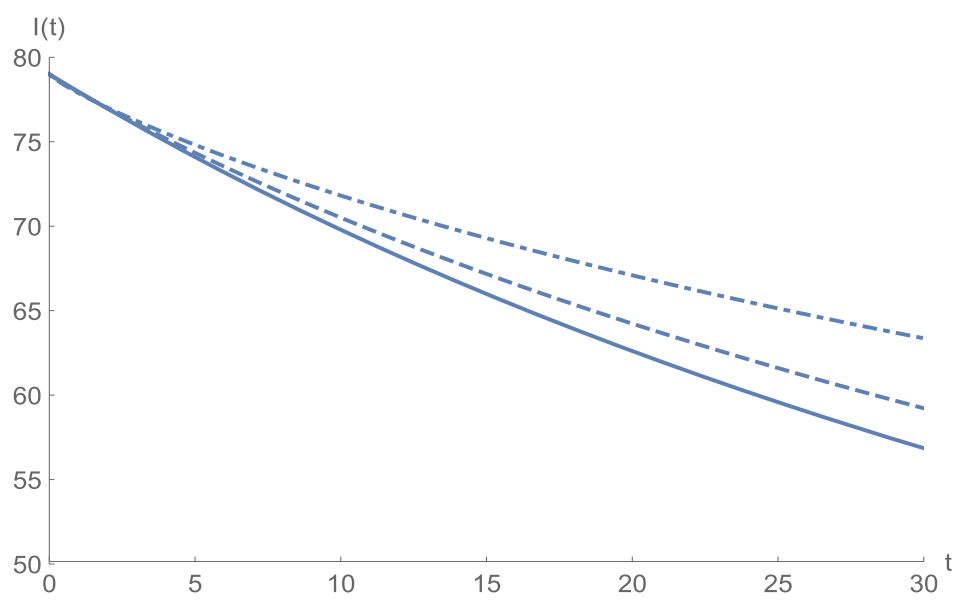

Figure $8 I(t)$ against $t$ : (solid line) $a=1.0$, (dot-dashed line) $a=0.85$, (dashed line) $a=0.75$ 


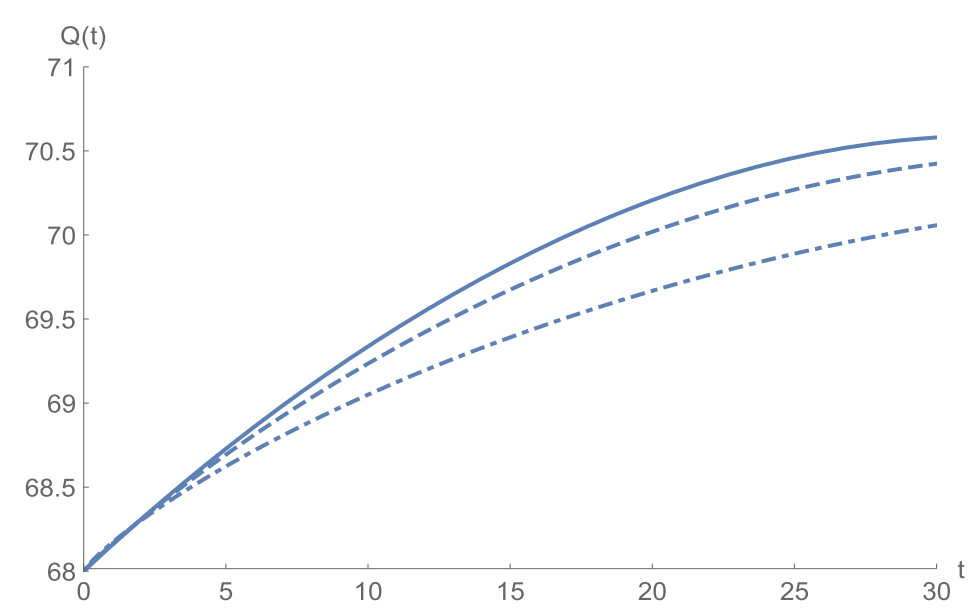

Figure $9 Q(t)$ against $t$ : (solid line) $a=1.0$, (dot-dashed line) $a=0.85$, (dashed line) $a=0.75$

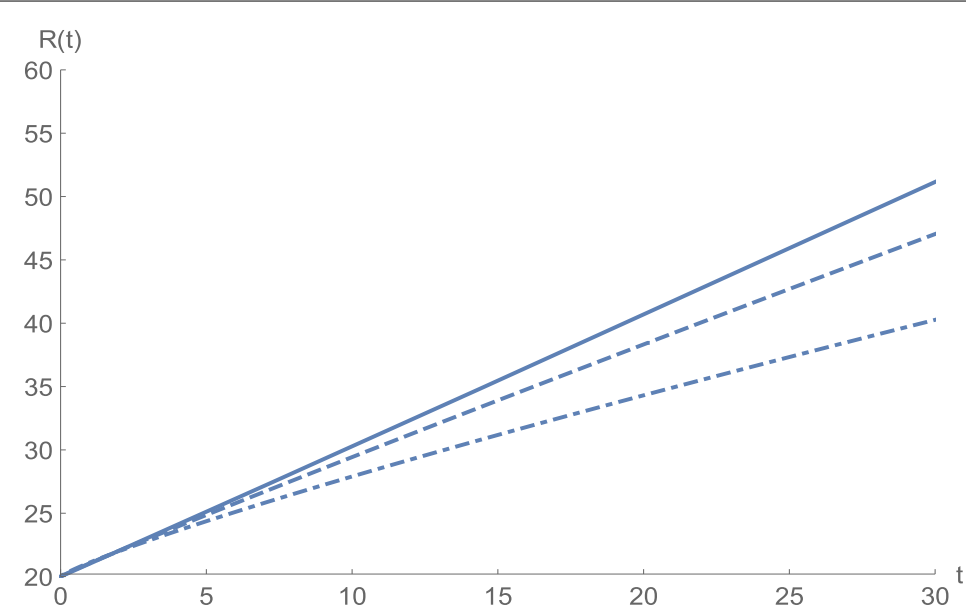

Figure $10 R(t)$ against $t$ : (solid line) $a=1.0$, (dot-dashed line) $a=0.85$, (dashed line) $a=0.75$

\section{Remarks and conclusions}

In this paper, we have formulated and analyzed a new mathematical model for COVID-19 epidemic. The model is described by a system of fractional-order differential equations and includes five classes, namely, $S$ (susceptible class), $E$ (exposed class), I (infected class), $Q$ (isolated class), and $R$ (recovered class). It should be emphasized that the model is a generalization of our recent work proposed in [35].

Firstly, the positivity, boundedness, and stability of the model have been established. Furthermore, the basic reproduction number of the model has been calculated by using the next generation matrix approach. Lastly, we have applied the adaptive predictor-corrector algorithm and fourth-order Runge-Kutta (RK4) method to simulate the proposed model. A set of numerical simulations has been performed to support the validity of the theoretical results. The numerical simulations indicate that there is a good agreement between theoretical results and numerical ones. 
In the near future, we will extend the results in this work to propose new mathematical models for COVID-19 epidemic. Especially, effective strategies to control and prevent the disease will be investigated.

\section{Acknowledgements}

The authors thank the editor and anonymous referees for useful comments that led to a great improvement of the paper.

\section{Funding}

This paper was supported by the Natural Science Foundation of the Higher Education Institutions of Anhui Province (No. KJ2020A0002)

\section{Availability of data and materials}

The authors confirm that the data supporting the findings of this study are available within the article cited herein.

\section{Competing interests}

The authors declare that there is no conflict of interest regarding the publication of this paper.

\section{Authors' contributions}

Authors have equally contributed in preparing this manuscript. All authors read and approved the final manuscript.

\section{Author details}

${ }^{1}$ School of Management Science and Engineering, Anhui University of Finance and Economics, Bengbu, 233030, China. 2 Department of Mathematics, COMSATS University Islamabad, Abbottabad Campus, Abbottabad, 22060, Khyber Pakhtunkhwa, Pakistan. ${ }^{3}$ Division of Clinical Pharmacology, Department of Medicine, University of Cape Town, Cape Town, South Africa. ${ }^{4}$ Department of Mathematics, Faculty of Arts and Sciences, Ondokuz Mays University, 55139, Samsun, Turkey.

\section{Publisher's Note}

Springer Nature remains neutral with regard to jurisdictional claims in published maps and institutional affiliations.

Received: 31 May 2020 Accepted: 31 July 2020 Published online: 14 August 2020

\section{References}

1. Abdo, M.S., Hanan, K.S., Satish, A.W., Pancha, K.: On a comprehensive model of the novel coronavirus (COVID-19) under Mittag-Leffler derivative. Chaos Solitons Fractals 135, 109867 (2020)

2. Atangana, A.: Fractal-fractional differentiation and integration: connecting fractal calculus and fractional calculus to predict complex system. Chaos Solitons Fractals 102, 396-406 (2017). https://doi.org/10.1016/j.chaos.2017.04.027

3. Atangana, A.: Blind in a commutative world: simple illustrations with functions and chaotic attractors. Chaos Solitons Fractals 114, 347-363 (2018). https://doi.org/10.1016/j.chaos.2018.07.022

4. Atangana, A.: Fractional discretization: the African's tortoise walk. Chaos Solitons Fractals 130, 109399 (2020). https://doi.org/10.1016/j.chaos.2019.109399

5. Bekiros, S., Kouloumpou, D.: SBDiEM: a new mathematical model of infectious disease dynamics. Chaos Solitons Fractals 136, $109828(2020)$

6. Bocharov, G., Volpert, V., Ludewig, B., Meyerhans, A.: Mathematical Immunology of Virus Infections. Springer, Berlin (2018)

7. Brauer, F.: Mathematical epidemiology: past, present, and future. Infect. Dis. Model. 2, 113-127 (2017)

8. Brauer, F., van den Driessche, P., Wu, J.: Mathematical Epidemiology. Springer, Berlin (2008)

9. Cakan, S.: Dynamic analysis of a mathematical model with health care capacity for COVID-19 pandemic. Chaos Solitons Fractals 139, 110033 (2020)

10. Caputo, M.: Linear models of dissipation whose $Q$ is almost frequency independent-II. Geophys. J. Int. 13(5), 529-539 (1967). https://doi.org/10.1111/j.1365-246X.1967.tb02303.x

11. Daftardar-Gejii, V.: Fractional Calculus and Fractional Differential Equations. Birkhäuser, Basel (2019)

12. Delavari, H., Baleanu, D., Sadati, J.: Stability analysis of Caputo fractional-order nonlinear systems revisited. Nonlinear Dyn. 67, 2433-2439 (2012)

13. Diethelm, K., Ford, N., Freed, A.: A predictor-corrector approach for the numerical solution of fractional differential equations. Nonlinear Dyn. 29, 3-22 (2002)

14. Higazy, M.: Novel fractional order SIDARTHE mathematical model of COVID-19 pandemic. Chaos Solitons Fractals $138,110007(2020)$

15. Kumar, S., Cao, J., Abdel-Aty, M.: A novel mathematical approach of COVID-19 with non-singular fractional derivative. Chaos Solitons Fractals 139, 110048 (2020)

16. Li, Y., Cheng, Y., Podlubny, I.: Mittag-Leffler stability of fractional order nonlinear dynamic systems. Automatica 45 1965-1969 (2009)

17. Lin, W.: Global existence theory and chaos control of fractional differential equations. J. Math. Anal. Appl. 332(1) 709-726 (2007). https://doi.org/10.1016/j.jmaa.2006.10.040

18. Martcheva, M.: An Introduction to Mathematical Epidemiology. Springer, New York (2015)

19. Ming, W., Huang, J.V., Zhang, C.J.P.: Breaking down of the healthcare system: mathematical modelling for controlling the novel coronavirus (2019-nCoV) outbreak in Wuhan, China (2020). https://doi.org/10.1101/2020.01.27.922443

20. Odibat, Z., Baleanu, D.: Numerical simulation of initial value problems with generalized Caputo-type fractional derivatives. Appl. Numer. Math. 156, 94-110 (2020) 
21. Odibat, Z., Baleanu, D.: Numerical simulation of initial value problems with generalized Caputo-type fractional derivatives. Appl. Numer. Math. 156, 94-105 (2020). https://doi.org/10.1016/j.apnum.2020.04.015

22. Odibat, Z., Shawagfeh, N.: Generalized Taylor's formula. Appl. Math. Comput. 186(1), 286-293 (2007). https://doi.org/10.1016/j.amc.2006.07.102

23. Okuonghae, D., Omame, A.: Analysis of a mathematical model for COVID-19 population dynamics in Lagos, Nigeria. Chaos Solitons Fractals 139, 110032 (2020)

24. Oldham, K., Spanier, J.: The Fractional Calculus Theory and Applications of Differentiation and Integration to Arbitrary Order. Elsevier, Amsterdam (1974)

25. Podlubny, I.: Fractional Differential Equations. Academic Press, San Diego (1998)

26. Postnikov, E.B.: Estimation of COVID-19 dynamics "on a back-of-envelope": does the simplest SIR model provide quantitative parameters and predictions? Chaos Solitons Fractals 135, 109841 (2020)

27. Samko, S.G., Kilbas, A.A., Mariuchev, O.l.: Fractional Integrals and Derivatives. Taylor \& Francis, Milton Park (1993)

28. Shah, K., Abdeljawad, T., Mahariq, I., Jarad, F.: Qualitative analysis of a mathematical model in the time of COVID-19. BioMed Res. Int. 2020, Article ID 5098598 (2020). https://doi.org/10.1155/2020/5098598

29. Torrealba-Rodriguez, O., Conde-Gutiérrez, R.A., Hernández-Javiera, A.L.: Modeling and prediction of COVID-19 in Mexico applying mathematical and computational models. Chaos Solitons Fractals 138, 109946 (2020)

30. Ud Din, R., Shah, K., Ahmad, I., Abdeljawad, T.: Study of transmission dynamics of novel COVID-19 by using mathematical model. Adv. Differ. Equ. 2020, 323 (2020)

31. van den Driessche, P., Watmough, J.: Reproduction numbers and sub-threshold endemic equilibria for compartmental models of disease transmission. Math. Biosci. 180, 29-48 (2002)

32. Vargas-De-Leon, C.: Volterra-type Lyapunov functions for fractional-order epidemic systems. Commun. Nonlinear Sci. Numer. Simul. 24, 75-85 (2015)

33. Yousaf, M., Muhammad, S.Z., Muhammad, R.S., Shah, H.K.: Statistical analysis of forecasting COVID-19 for upcoming month in Pakistan. Chaos Solitons Fractals 138, 109926 (2020)

34. Zaman, G., Jung, H., Torres, D.F.M., Zeb, A.: Mathematical modeling and control of infectious diseases. Comput. Math. Methods Med. 2017, Article ID 7149154 (2017)

35. Zeb, A., Alzahrani, E., Erturk, V.S., Zaman, G.: Mathematical model for coronavirus disease 2019 (COVID-19) containing isolation class. BioMed Res. Int. 2020, Article ID 3452402 (2020). https://doi.org/10.1155/2020/3452402

36. Zhang, Z:: A novel COVID-19 mathematical model with fractional derivatives: singular and nonsingular kernels. Chaos Solitons Fractals 139, 110060 (2020). https://doi.org/10.1016/j.chaos.2020.110060

\section{Submit your manuscript to a SpringerOpen ${ }^{\circ}$ journal and benefit from:}

- Convenient online submission

$\checkmark$ Rigorous peer review

- Open access: articles freely available online

- High visibility within the field

Retaining the copyright to your article

Submit your next manuscript at $>$ springeropen.com 